\title{
Comparison of surgical conditions during propofol or desflurane anesthesia for endoscopic sinus surgery
}

\author{
Kwangrae Cho ${ }^{1}$, Jin Young Lee ${ }^{1}$, Sung Kuk Park ${ }^{2}$, Soon Ho Cheong ${ }^{1,3}$, Kun Moo Lee ${ }^{1}$, Se Hun Lim , \\ Jeong Han Lee ${ }^{1}$, Myoung-Hun Kim ${ }^{1}$, Hyun-Tae Kim ${ }^{1}$, and Jung-Kyu Park ${ }^{1}$ \\ Departments of ${ }^{1}$ Anesthesiology and Pain Medicine, ${ }^{2}$ Otorhinolaryngology, Busan Paik Hospital, College of Medicine, Inje University, \\ ${ }^{3}$ Paik Institute for Clinical Research, Inje University, Busan, Korea
}

Background: Reduction of intraoperative bleeding is necessary to achieve the ideal surgical field for the endoscopic sinus surgery (ESS). Intraoperative intra nasal bleeding is influenced by various anesthetics. This study compared surgical field condition between propofol/remifentanil (PR) based anesthesia and desflurane/remifentanil (DR) based anesthesia.

Methods: American Society of Anesthesiologists physical status class I or II patients undergoing ESS were randomly assigned to group PR $(\mathrm{n}=36)$ or group DR $(\mathrm{n}=32)$. The extent of the preoperative surgical lesion was classified as high ( $>12)$ and low ( $\leq 12)$ Lund-Mackay (LM) scores according to the computed tomography findings. The target mean blood pressure was maintained at $70-80 \mathrm{mmHg}$. Only one surgeon was involved in rating the visibility of the surgical field on a numeric rating scale (NRS) every 10 minutes.

Results: There was a different surgical field grade from PR to DR. The mean (SD) surgical field score of NRS for the PR and DR was $2.3(0.57)$ and $2.7(0.67)$, respectively $(\mathrm{P}=0.006)$. Especially in the high-LM score patients, the mean (SD) of surgical field score for the PR and DR was $2.4(0.67)$ and $3.0(0.63)$, respectively $(\mathrm{P}=0.012)$.

Conclusions: In the high-LM score patients, PR based anesthesia resulted in better surgical field condition for ESS than DR based anesthesia. In ESS, PR based anesthesia is considered to be helpful. (Korean J Anesthesiol 2012; 63: 302-307)

Key Words: Desflurane, Propofol, Remifentanil, Sinusitis.

Received: January 30, 2012. Revised: April 12, 2012. Accepted: May 15, 2012.

Corresponding author: Kwangrae Cho, M.D., Department of Anesthesiology and Pain Medicine, Busan Paik Hospital, Gaegeum 2-dong, Busanjin-gu, Busan 614-735, Korea. Tel: 82-51-890-6520, Fax: 82-51-898-4216, E-mail: maeteo@hanmir.com This is a thesis for a master's degree.

(c) This is an open-access article distributed under the terms of the Creative Commons Attribution Non-Commercial License (http:// creativecommons.org/licenses/by-nc/3.0/), which permits unrestricted non-commercial use, distribution, and reproduction in any medium, provided the original work is properly cited. 


\section{Introduction}

Endoscopic sinus surgery (ESS) is considered a selective treatment method and is widely performed in chronic sinusitis patients who do not respond to drug treatment. ESS can be performed under local anesthesia, but in most cases, it is performed under general anesthesia.

Intra-nasal bleeding during ESS surgery under general anesthesia makes the visible surgical field difficult; therefore bleeding can lengthen the time of the surgery and increase complications. Methods to reduce intra-nasal bleeding, such as keeping the head elevated during surgery to reduce intra nasal bleeding, nasal wall epinephrine administration [1], bradycardia that is induced during surgery [2], and inducing low blood pressure through fast-acting $\beta$-blocker or magnesium administration during surgery [3] or $\alpha$-agonist administration before surgery $[4,5]$. There is also the method of reducing intra nasal bleeding to obtain a visible surgical field through the selection of anesthesia.

In a direct comparison study of intravenous anesthesia such as propofol and inhalation anesthesia such as isoflurane and sevoflurane, intravenous anesthesia caused less intra nasal bleeding [6-8]. In another study that used the LundMackay (LM) score from computed tomography (CT) findings to compare the extent of sinusitis and anesthesia in a severe sinusitis patient group, less bleeding and a better visible surgical field was noted in the group that received intravenous anesthetic with propfol than the group that received sevoflurane inhalation anesthesia [9].

This study attempted to compare the surgical field scores, according to the extent of the preoperative surgical lesions, for a group that used propofol/remifentanil (PR), reported to reduce the amount of bleeding to obtain a visible surgical field to a group that used sevoflurane/remifentanil, and to a group that used desflurane/remifentanil (DR).

\section{Materials and Methods}

Sixty-eight patients with the American Society of Anesthesiologists physical status class I or II diagnosed with chronic sinusitis and scheduled to receive endoscopic sinus surgery were chosen as subjects. Ahn et al. [9] noted that a numeric rating scale (NRS) was 4.8 on average and the standard deviation 2.4 when sevoflurane was utilized. In case of desflurane, a NRS was 2.9 on average, and standard deviation 1.6. We expected a conclusion similar to these, it was estimated that 32 subjects were needed to give this study a statistical power of $80 \%$ show a clinically relevant, a significance level of 0.05 . As we expected $10 \%$ dropouts, we included 36 subjects in the each group. In this period, we had 32 patients in the DR group,
36 patients in the PR group. So, we performed a statistical analysis with these patients. This study attained approval from the clinical ethics board of the hospital. The patients were visited before anesthesia was given and the purpose of the study and possible complications were explained the written consent was obtained. The 68 patients were randomly assigned to two groups, PR $(\mathrm{n}=36)$ and DR $(\mathrm{n}=32)$. Patients taking antiplatelet agents or anticoagulants, patients with other bleeding tendencies, patients with asthma or fungal sinusitis, and patients undergoing surgery again were excluded from the study. To investigate the extent of the preoperative surgical lesion, the Lund-Mackay CT score was calculated [10] (Table 1), and a score $>12$ was categorized as a high-LM score and $\leq 12$ as a low-LM score.

There were no medications before anesthesia, and basic noninvasive monitoring of arterial pressure, electrocardiogram, and pulse oximetry were initiated upon arrival into the operating room. The blood pressure was checked every 3 minutes. Anesthesia was induced with lidocaine $0.5 \mathrm{mg} / \mathrm{kg}$, propofol $2 \mathrm{mg} / \mathrm{kg}$, and rocuronium $0.6 \mathrm{mg} / \mathrm{kg}$ for both groups. Two $\mathrm{L} / \mathrm{min}$ of air- $\mathrm{O}_{2}$ was used to administer $0.5 \mathrm{FiO}_{2}$. Desflurane was administered at a 6-9 vol\% for the DR group. For the PR group, propofol was continually injected using target a concentration infusion (TCI, Orchestra ${ }^{\circledR}$ Base Primea, Fresenius kabi, Germany) where the target blood concentration was set as $2-6 \mu \mathrm{g} / \mathrm{ml}$. Both groups were equally intravenous injected with remifentanil $0.2 \mu \mathrm{g} / \mathrm{min} /$ $\mathrm{kg}$ during anesthesia induction, and after inducing anesthesia, it was continually injected at a maintenance dose of $0.15 \mu \mathrm{g} /$ $\mathrm{min} / \mathrm{kg}$.

Table 1. Lund-Mackay Computed Tomography Staging System

\begin{tabular}{|c|c|c|c|}
\hline & $\begin{array}{c}\text { No } \\
\text { abnormality }\end{array}$ & $\begin{array}{c}\text { Partial } \\
\text { opacification }\end{array}$ & $\begin{array}{c}\text { Total } \\
\text { opacification }\end{array}$ \\
\hline \multicolumn{4}{|l|}{ Anterior ethmoid } \\
\hline $\mathrm{R}$ & 0 & 1 & 2 \\
\hline $\mathrm{L}$ & 0 & 1 & 2 \\
\hline \multicolumn{4}{|c|}{ Posterior ethmoid } \\
\hline $\mathrm{R}$ & 0 & 1 & 2 \\
\hline $\mathrm{L}$ & 0 & 1 & 2 \\
\hline \multicolumn{4}{|l|}{ Maxillary } \\
\hline $\mathrm{R}$ & 0 & 1 & 2 \\
\hline $\mathrm{L}$ & 0 & 1 & 2 \\
\hline \multicolumn{4}{|l|}{ Frontal } \\
\hline $\mathrm{R}$ & 0 & 1 & 2 \\
\hline $\mathrm{L}$ & 0 & 1 & 2 \\
\hline \multicolumn{4}{|l|}{ Sphenoid } \\
\hline $\mathrm{R}$ & 0 & 1 & 2 \\
\hline $\mathrm{L}$ & 0 & 1 & 2 \\
\hline $\begin{array}{l}\text { Ostiomeatal } \\
\text { complex }\end{array}$ & $\begin{array}{c}\text { Non- } \\
\text { obstructed }\end{array}$ & & Obstructed \\
\hline $\mathrm{R}$ & 0 & & 2 \\
\hline $\mathrm{L}$ & 0 & & 2 \\
\hline
\end{tabular}


The target blood pressure was maintained at a mean arterial pressure of 70-80 $\mathrm{mmHg}$, and was first controlled with a desflurane or propofol concentration. When blood pressure was not controlled with these two drugs, the concentration of remifentanil was controlled within $0.06-0.2 \mu \mathrm{g} / \mathrm{min} / \mathrm{kg}$ to maintain the target blood pressure. End-tidal $\mathrm{CO}_{2}$ concentration was $35-40 \mathrm{mmHg}$, and a reverse Trendelenburg position of $20^{\circ}$ was maintained. An infusion pump with a syringe filled with propofol was also mounted for the subjects in the DR group so a single blind experiment was conducted and the operating surgeon was blinded to the type of anesthesia that was used.

For all surgeries, a surgeon injected mixed solution of epinephrine and lidocaine 0.3 cc (1 : 100,000 epinephrine: lidocaine $2 \%$ at $1: 1$ ) at the both origin of middle turbinates. For all surgeries, in 10 minutes intervals, the same operating surgeon measured the surgical field grade, which was expressed in a NRS of $0-5$ [11] (Table 2). The precise amount of blood loss could not be measured, but hemoglobin figures before and directly after surgery were measured to investigate the difference.

Statistical analysis was done with SPSS (version 18.0, SPSS Incorporation, Chicago, IL, USA). Each category was expressed in the form of the mean (SD), and comparison analysis was done with Student's t-test and Chi-square test. It was considered statistically significant when the P value was less than 0.05 .

\section{Results}

There were no statistical differences in the demographic characteristics or in the extent of sinusitis between the two groups (Table 3). There were also no statistical differences in the operating time, mean remifentanil infusion rate, amount of fluid administered during surgery, mean arterial pressure, and heart rate and in hemoglobin figures measured before and after surgery between the two groups (Table 4).

The NRS of the surgical field grade was lower in the PR group than in the DR group (2.3 [0.57] vs 2.7 [0.67], P = 0.006). Twentyone patients in the PR group and seventeen patients in the DR group exhibited high-LM scores, while 15 in the PR group and 15 in the DR group exhibited low-LM scores. In the high-LM score, the surgical field grade, NRS, was lower in the PR group than in the DR group (2.4 [0.67] vs 3.0 [0.63], $\mathrm{P}=0.012)$, but there were no statistical differences between the PR group and DR group in the low-LM score $(2.4[0.56]$ vs 2.2 [0.42], $\mathrm{P}=0.861)$ (Table 5).

The NRS of the surgical field grade was significantly higher in the high-LM score patients than in the low-LM score patients in the DR group.

Table 2. Surgical Grade Scoring System Designed Specifically for Use in Endoscopic Sinus Surgery

\begin{tabular}{cl}
\hline Grade & \multicolumn{1}{c}{ Assessment } \\
\hline 0 & No bleeding (cadaveric conditions) \\
1 & Slight bleeding - no suctioning required \\
2 & Slight bleeding - occasional suctioning required \\
3 & Slight bleeding - frequent suctioning required; bleeding threatens surgical field a few seconds after suction is removed \\
4 & Moderate bleeding - frequent suctioning required and bleeding threatens surgical field directly after suction is removed \\
5 & Severe bleeding - constant suctioning required; bleeding appears faster than can be removed by suction; surgical field severely \\
& threatened and surgery usually not possible \\
\hline
\end{tabular}

Table 3. Demographic and Clinical Characteristics of Patients

\begin{tabular}{lccc}
\hline & Group DR $(\mathrm{n}=32)$ & Group PR $(\mathrm{n}=36)$ & P value \\
\hline Sex $(\mathrm{M} / \mathrm{F})$ & $17 / 15$ & $18 / 18$ & 0.816 \\
Age $(\mathrm{yr})$ & $41.7(15.3)$ & $43.5(15.6)$ & 0.643 \\
Height $(\mathrm{cm})$ & $167.7(9.0)$ & $164.8(8.1)$ & 0.166 \\
Weight $(\mathrm{kg})$ & $67.2(13.4)$ & $62.8(10.2)$ & 0.138 \\
Body mass index $\left(\mathrm{kg} / \mathrm{m}^{2}\right)$ & $23.7(3.4)$ & $23.1(2.9)$ & 0.372 \\
ASA classification $(\mathrm{I} / \mathrm{II})$ & $24 / 8$ & $29 / 7$ & 0.770 \\
Total LM score & $13.3(5.2)$ & $13.1(4.5)$ & 0.808 \\
Patient of high/low LM score & $17 / 15$ & $21 / 15$ & 0.807
\end{tabular}

The values are reported as the mean (SD) or the number of patients. Group DR: desflurane/remifentanil group, Group PR: propofol/remifentanil group, ASA: American Society of Anesthesiologists physical status, LM: Lund-Mackay, High LM score: LM score > 12, Low LM score, LM score $\leq 12$. 
Table 4. Intraoperative Variables

\begin{tabular}{lccc}
\hline & Group DR & Group PR & P value \\
\hline Duration of surgery (min) & $31.8(8.9)$ & $32.8(10.7)$ & 0.716 \\
Average endtidal concentration of desflurane $(\%)$ & $6.6(1.16)$ & - & - \\
Average concentration of propofol at effect site $(\mu \mathrm{g} / \mathrm{ml})$ & - & $5.2(0.8)$ & - \\
Average rate of remifentanil infusion $(\mu \mathrm{g} / \mathrm{kg} / \mathrm{min})$ & $0.16(0.04)$ & $5.17(0.04)$ & 0.163 \\
Average intraoperative fluid administration $(\mathrm{ml} / \mathrm{kg} / \mathrm{hr})$ & $5.3(1.0)$ & $73.3(4.3)$ & 0.673 \\
Intraoperative MAP $(\mathrm{mmHg})$ & $72.5(3.7)$ & $65.3(10.0)$ & 0.425 \\
Intraoperative HR (beats/min) & $66.2(8.5)$ & $0.7(0.4)$ & 0.684 \\
Difference of Hgb $(\mathrm{g} / \mathrm{dl})$ & $0.9(0.6)$ & 0.152 \\
\hline
\end{tabular}

The values are reported as the mean (SD) or the number of patients. Group DR: desflurane/remifentanil group, Group PR: propofol/remifentanil group, MAP: mean arterial pressure, HR: heart rate, Difference of Hgb: the value taking out postoperative hemoglobin figure from preoperative hemoglobin figure.

Table 5. Numeric Rating Scale of Surgical Field Grade

\begin{tabular}{|c|c|c|c|}
\hline \multirow{2}{*}{ Surgical condition } & \multicolumn{3}{|c|}{ Group } \\
\hline & Group DR & Group PR & $P$ value \\
\hline Average numeric rating scale of surgical field grade & $2.7(0.67) *(\mathrm{n}=32)$ & $2.3(0.57)(\mathrm{n}=36)$ & 0.006 \\
\hline High LM & $3.0(0.63)^{\dagger}(\mathrm{n}=17)$ & $2.4(0.67)(\mathrm{n}=21)$ & 0.012 \\
\hline Low LM & $2.4(0.56)(\mathrm{n}=15)$ & $2.2(0.42)(\mathrm{n}=15)$ & 0.861 \\
\hline
\end{tabular}

The values are reported as the mean (SD) or the number of patients. Group DR: desflurane/remifentanil group, Group PR: propofol/ remifentanil group, LM: Lund-Mackay, High LM score: LM score $>12$, Low LM score: LM score $\leq 12 .{ }^{*} \mathrm{P}<0.05$ compared to Group PR, ${ }^{\dagger} \mathrm{P}<0.05$ compared to High LM of Group PR and Low LM of Group DR and PR.

\section{Discussion}

In the results of this study, the intravenous anesthesia group using propofol/remifentanil provided better visibility for the surgical field than the inhalation anesthesia group using desflurane/remifentanil. The visual status was especially good in the intravenous anesthesia group in the high-LM score patients.

Desflurane is a representative inhalation anesthesia that has been clinically used since it was first introduced in 1992. Desflurane produces two distinct cardiovascular actions [12]. First, desflurane decreases left ventricular systolic and diastolic function. It decreases systemic vascular resistance and mean arterial blood pressure in a dose-dependent fashion. Heart rate is unchanged at lower steady-state concentrations, but increases with higher concentrations. Second, when endtidal concentration rapidly increases to more than 1 minimum alveolar concentration, it accelerates the sympathetic nerve system to increase the heart rate and mean arterial pressure. In our study, these characteristics of desflurane were taken consideration, and the flow was controlled at $2 \mathrm{~L} / \mathrm{min}$ to prevent a rapid increase in alveolar concentration.

Propofol is a representative intravenous anesthesia which causes a decrease in the mean arterial pressure by reducing cardiac output and systemic vascular resistance. However it has the characteristic where the heart rate increases according to the fall in blood pressure, which is slowed down due to the suppression of the baroreflex [13].

Remifentanil reduces the mean arterial pressure by reducing the cardiac output without reducing systemic vascular resistance; thus, it generally reduces capillary bleeding in ESS and improves visibility during surgery [14]. Inhalation anesthesia, intravenous anesthesia, and opioids all suppress the cardiovascular relationship to reduce the mean arterial pressure, but the mechanism in which the mean arterial pressure is reduced shows differences according to each drug. Inhalation anesthesia largely reduces the arterial pressure according to the reducing systemic vascular resistance; thus, despite low blood pressure, capillary bleeding increases [15]. Propofol also reduces the vascular resistance, but there is difference between inhalation agents to propofol. In contrast, propofol may either reset or inhibit the baroreflex, reducing the tachycardiac response to hypotension from decreased systemic vascular resistance [16]. In addition, when propofol is continually infused, it can cause a reduction in the cerebral perfusion pressure, which regulates the cerebral blood flow [17]. The ethmoid, sphenoid, frontal sinuses are supplied with blood by the branches of internal carotid artery: the ethmoidal and supraorbital artery [18]. The internal carotid artery is a division of cerebral circulation. Therefore, reduced bleeding can be expected due to the reduced blood flow around the paranasal sinuses.

As can be seen, when comparing inhalation anesthesia and 
intravenous anesthesia, there are differences in the mechanism by which the mean arterial pressure is reduced, and each drug has its own characteristics.

As a method to evaluate the extent of chronic sinusitis, there is the Lund-Mackay CT staging system. In contrast to other staging systems, the Lund-Mackay staging system introduces a point system instead of stages therefore, when other staging systems have around 4 stages, the Lund-Mackay can have up to 25 stages from 0 point to 24 points, which had the effect of being able to specify small differences in lesions. In addition, the points were simplified to 0 point (normal), 1 point (partially corrupt), and 2 points (completely corrupt), which heightened the concordance within observers and between observers. Lund and Mackay [10] reported that there was a $98 \%$ concordance between two observers in 200 cases, and emphasized the system's user friendliness through simplification. On the basis of the report that severity increases as LM score increases such as mild (0-6), moderate ( $7-12)$, moderately severe (13-18), and severe (19-24) [19], the LM score was categorized into low $(0-12)$ and high $(13-24)$. As the LM-score elevated, it means that the extent of sinusitis was severe and that there was increased vessel distribution in the operating area, which can be a predictive factor for the tendency of high bleeding [20]. Beule et al. [21] compared anesthesia using propofol and anesthesia using sevoflurane, and Yoo et al. [22] also compared anesthesia using propofol/remifentanil, anesthesia using sevoflurane/ remifentanil, and anesthesia using desflurane/remifentanil, but these studies did not consider the difference of the LM score, and thus, the results were that there were no differences between the anesthetic drugs. Ahn et al. [9] compared ESS in patients who received inhalation anesthesia of sevoflurane/ remifentanil and intravenous anesthesia of propofol/remifentanil, and reported that in low-LM scores, there was a similar visibility for the surgical field regardless of the anesthetic drug, but in high-LM score, there were significant differences according to the type of anesthesia used. Our study compared anesthesias using propofol/remifentanil and desflurane/ remifentanil, and also compared the difference according to the LM score. Our study revealed that in the low-LM score, a similar level of visibility was obtained regardless of anesthetic drug, but in the high-LM score, anesthesia using propofol/remifentanil had a better visibility of the surgical field compared to the anesthesia using desflurane/remifentanil.

Aside from this, many other factors affect the degree of bleeding during ESS. Therefore, this study tried to control for the other factors that may affect bleeding to investigate the difference according to the anesthetic drug. The mean arterial pressure, which mainly contributes to bleeding during surgery, was maintained at $70-80 \mathrm{mmHg}$, the lowest constraint of blood pressure which does not cause ischemia in the organs, and the pulse rate per minute was maintained around 70 during the surgery. Additionally, to compensate for the limitation of the study deducing results from subjective NRS, a single surgeon evaluated the visibility of the surgical field.

To remove personal error from the evaluation of the visible surgical field, an important result in our study would be to measure the actual blood loss. The authors did measure the difference in hemoglobin figures before and after surgery instead of actual blood loss. However, it is hard to determine an accurate amount of blood loss with these figures due to the effect of fluids being administered during surgery and the short operating time resulting in an insufficient hemoglobin compensation time. Like the study by Ahn et al. [9], the study is limited in the fact that the actual blood loss was not evaluated along with the visible surgical field. In addition, further research regarding the difference in the visible surgical field according to anesthesia and LM score should be conducted in a 3-way research with desflurane and sevoflurane, as in a study by Yoo et al. [22].

This study revealed that in ESS, patients with a high-LM score had a better visible surgical field for the operating surgeon when under intravenous anesthesia using propofol/remifentanil than under inhalation anesthesia using desflurane/remifentanil. It should be considered that using propofol/remifentanil for general anesthesia will be helpful when performing ESS.

\section{Acknowledgments}

This work was supported by Grant from Inje University, 2010.

\section{References}

1. Leigh JM. The history of controlled hypotension. Br J Anaesth 1975; 47: 745-9.

2. Nair S, Collins M, Hung P, Rees G, Close D, Wormald PJ. The effect beta-blocker premedication on the surgical field during endoscopic sinus surgery. Laryngoscope 2004; 114: 1042-6.

3. Elsharnouby NM, Elsharnouby MM. Magnesium sulphate as a technique of hypotensive anesthesia. Br J Anaesth 2006; 96: 727-31.

4. Okuyama K, Inomata S, Toyooka H. The effects of prostaglandin E1 or oral clonidine premedication on blood loss during paranasal sinus surgery. Can J Anaesth 2005; 52: 546-7.

5. Durmus M, But AK, Dogan Z, Yucei A, Miman MC, Ersoy MO. Effect of dexmedetomidine on bleeding during tympanoplasty or septorhinoplasty. Eur J Anaesthesiol 2007; 24: 447-53.

6. Dogan IV, Ovali E, Eti Z, Yayci A, Gogus FY. The in vitro effects of isoflurane, sevoflurane, and propofol on platelet aggregation. Anesth Analg 1999; 88: 432-6.

7. Tirelli G, Bigarini S, Russolo M, Lucangelo U, Gullo A. Total intravenous anaesthesia in endoscopic sinus-nasal surgery. Acta Otorhinolaryngol Ital 2004; 24: 137-44.

8. Manola M, De Luca E, Moscillo L, Mastella A. Using remifentanil 
and sufentanil in functional endoscopic sinus surgery to improve surgical conditions. ORL J Otorhinolaryngol Relat Spec 2005; 67: 836.

9. Ahn HJ, Chung SK, Dhong HJ, Kim HY, Ahn JH, Lee SM, et al. Comparison of surgical conditions during propofol or sevoflurane anaesthesia for endoscopic sinus surgery. Br J Anaesth 2008; 100: 50-4.

10. Lund VJ, Mackay IS. Staging in rhinosinusitus. Rhinology 1993; 31: 183-4.

11. Fromme GA, MacKenzie RA, Gould AB Jr, Lund BA, Offord KP. Controlled hypotension for orthognathic surgery. Anesth Analg 1986; 65: 683-6.

12. Weiskopf RB. Cardiovascular effects of desflurane in experimental animals and volunteers. Anaesthesia 1995; 50 Suppl: 14-7.

13. Ebert TJ, Muzi M, Berens R, Goff D, Kampine JP. Sympathetic responses to induction of anesthesia in humans with propofol or etomidate. Anesthesiology 1992; 76: 725-33.

14. Manola M, De Luca E, Moscillo L, Mastella A. Using remifentanil and sufentanil? in functional endoscopic sinus surgery to improve surgical condition. ORL J Otorhinolaryngol Relat Spec 2005; 67: 836.

15. Blackwell KE, Ross DA, Kapur P, Calcaterra TC. Propofol for maintenance of general anesthesia: a technic to limit blood loss during endoscopic sinus surgery. Am J Otolaryngol 1993; 14: 262-6.
16. Ebert TJ, Muzi M, Berens R, Goff D, Kampine JP. Sympathetic responses to induction of anesthesia in humans with propofol or etomidate. Anesthesiology 1992; 76: 725-33.

17. Haberer JP, Audibert G, Saunier CG, Muller C, Laxenaire MC, Hartemann D. Effect of propofol and thiopentone on regional blood flow in brain and peripheral tissues during normoxia and hypoxia in the dog. Clin Physiol 1993; 13: 197-207.

18. Činčikas D, Ivaškevičius J, Martinkènas JL, Balseris S. A role of anesthesiologist in reducing surgical bleeding in endoscopic sinus surgery. Medicina (Kaunas) 2010; 46: 730-4.

19. Rhyoo C, Jung MK, Lee JH. The clinical significance of LundMackay CT staging system in assessing the severity of chronic rhinosinusitis. Korean J Otolaryngol-Head Neck Surg 2001; 44: 83741.

20. Mortuaire G, Bahij J, Maetz B, Chevalier D. Lund-Mackay score is predictive of bleeding in ethmoidectomy for nasal polyposis. Rhinology 2008; 46: 285-8.

21. Beule AG, Wilhelmi F, Kühnel TS, Hansen E, Lackner KJ, Hosemann W. Propofol versus sevoflurane: bleeding in endoscopic sinus surgery. Otolaryngol Head Neck Surg 2007; 136: 45-50.

22. Yoo HS, Han JH, Park SW, Kim KS. Comparison of surgical condition in endoscopic sinus surgery using remifentanil combined with propofol, sevoflurane, or desflurane. Korean J Anesthesiol 2010; 59: 377-82. 